\title{
Quasimonoenergetic electron acceleration in the self-modulated laser wakefield regime
}

\author{
B. Hidding ${ }^{1}$ M. Geissler, ${ }^{2,3}$ G. Pretzler, ${ }^{1}$ K.-U. Amthor, ${ }^{4}$ H. Schwoerer, ${ }^{5}$ S. Karsch, ${ }^{3}$ \\ L. Veisz, ${ }^{3}$ K. Schmid, ${ }^{3,6}$ and R. Sauerbrey \\ ${ }_{1}^{1}$ Institut für Laser-und Plasmaphysik, Heinrich-Heine-Universität Düsseldorf, 40225 Düsseldorf, Germany \\ ${ }^{2}$ Department of Physics and Astronomy, Centre for Plasma Physics, Queen's University Belfast, \\ University Road, Belfast BT7 1NN, Northern Ireland, United Kingdom \\ ${ }^{3}$ Max-Planck-Institut für Quantenoptik, 85748 Garching, Germany \\ ${ }_{5}^{4}$ Institut für Optik und Quantenelektronik, Friedrich-Schiller-Universität Jena, 07743 Jena, Germany \\ ${ }^{5}$ Laser Research Institute, University of Stellenbosch, Stellenbosch 7600, South Africa \\ ${ }^{6}$ Department für Physik, Ludwig-Maximilians-Universität München, 85748 Garching, Germany \\ ${ }^{7}$ Forschungszentrum Dresden-Rossendorf, 01328 Dresden, Germany
}

(Received 23 January 2009; accepted 6 March 2009; published online 14 April 2009)

\begin{abstract}
Details on the generation of (multiple) quasimonoenergetic electron bunches in the self-modulated laser wakefield acceleration (SMLWFA) regime are presented. This type of laser-plasma interaction can result in pronounced longitudinal laser pulse fragmentation, dependent on plasma density and laser intensity. It is shown by experiments and particle-in-cell simulations that these laser pulse fragments can be powerful enough to trigger nonlinear plasma wave breaking, injection, and acceleration of electrons to quasimonoenergetic energies. With high plasma densities, selfmodulation is promoted, and the advantages of SMLWFA such as especially high accelerating fields and short electron bunches $(<5 \mathrm{fs})$ can be harvested. In addition, more than one quasimonoenergetic electron bunch can be created, with a temporal spacing between each bunch of only few tens of femtoseconds, again governed by plasma density. (C) 2009 American Institute of Physics.

[DOI: $10.1063 / 1.3109666$ ]
\end{abstract}

\section{INTRODUCTION}

The energy a charged particle can gain in an accelerator is given as $W=q E d$, where $q$ is the charge, and $E$ is the averaged electric field the particle experiences over the acceleration distance $d$. Mainly because the electric fields $E$ in conventional accelerators are limited to few tens of $\mathrm{MV} / \mathrm{m}$, the demand for higher and higher particle energies could be satisfied for the most part only by introducing ever longer acceleration distances $d$, which led to the construction of machines as large as the large hadron collider.

Another approach was put up to discussion half a century ago, namely, to use the collective fields ${ }^{1-3}$ provided by temporary electron-ion separation in longitudinal plasma waves. ${ }^{4,5}$ These can be about four orders of magnitude stronger and thus, in principle, allow similarly powerful accelerator constructs on the submeter scale. Laser pulses focused to high intensities can effectively excite such plasma waves and the "Laser Electron Accelerator" was proposed about 30 years ago. ${ }^{6}$ Since then, both rapidly evolving laser techniques as well as scheme variations have finally led to the first quasimonoenergetic laser-plasma-produced electron bunches less than 5 years ago. ${ }^{7-9}$ The production of such quasimonoenergetic electron bunches can be descriptively explained by the bubble acceleration picture. ${ }^{10}$ Here, the electric field of the driver pulse, which propagates through the plasma with approximately the velocity of light $c$, is so intense that it pushes the plasma electrons out of its way (thus resembling electron cavitation as in Ref. 11 or electron blowout as in Ref. 12). When electrons swing back to the axis due to the attractive force of the (nearly) immobile ion background after the plasma period $\tau_{p}=\lambda_{p} / c$ (where $\lambda_{p}$ is the plasma wavelength), most electrons overshoot and oscillate on trajectories which are damped by collisional heating. In case the driver is longer than the plasma wavelength, the trajectories are even more complicated because then the electrons driven outward do not oscillate back completely but interact strongly with the repulsive electric force of the driver. In the ideal case, when the laser pulse is substantially shorter than the plasma wavelength, a blown out, electroncavitated bubble with the diameter of the plasma wavelength forms in the wakefield behind the driver pulse front. Electrons can be injected into and accelerated in the nonlinear field structure of the bubble, which is more pronounced the more effective the electron blowout becomes. Electron injection and plasma wave breaking can be a complicated process $^{13}$ even in the idealized case of a one-dimensional linear plasma wave, where due to the thermal electron energy distribution in the laser-heated plasma there is always a small fraction of electrons moving with a forward momentum high enough to be injected in the accelerating phase of the collective plasma wave field. This leads to the production of electron beams with exponential energy distributions and comparably big opening angles. With highly nonlinear breaking plasma waves present in the bubble regime, all the accelerated electrons are injected from approximately the same lateral position on axis at the vertex of the bubble, which moves along with the laser pulse during the interaction process. They experience the same accelerating and focusing three-dimensional field structure ${ }^{14}$ inside the bubble and therefore can leave the finite plasma target as ultrashort, 
quasimonoenergetic electron bunches with small divergences of a few milliradians behind the laser-plasma interaction zone.

Before the availability of sufficiently short and intense driving laser pulses and the discovery of the quasimonoenergetic bubble acceleration process, the so-called selfmodulated laser wakefield acceleration (SMLWFA) was in the focus of research. In contrast to laser wakefield acceleration (LWFA), where the laser pulse is shorter than the plasma period $\tau_{L}<\tau_{p}$, SMLWFA works with longer laser pulses and/or higher plasma densities, such that $\tau_{L}>\tau_{p}$. Under certain conditions, in SMLWFA the laser pulse breaks up into fragments with a distance $\approx \lambda_{p}$ during the interaction with the growing plasma wave. Due to the high plasma density, the accelerating wakefield can be especially high, scaling (in case of a linear plasma wave) as $E_{z} \propto n_{e}^{1 / 2}$. Based on this scheme, longitudinal electric fields of $E_{z}>200 \mathrm{GV} / \mathrm{m}$ (Ref. $15)$ and the generation of electron beams with energies up to $200 \mathrm{MeV}$ (Ref. 16) have been demonstrated several years ago. Another key advantage of SMLWFA results from the fact that the accelerating structures can be extremely small (due to the high densities, in the micrometer range) and the electron bunches accelerated inside these structures can thus be especially short $(<10 \mathrm{fs})$. On the other hand, the high plasma densities lead to enhancement of various instabilities, which can dominate the process.

Here, we present details on the generation of quasimonoenergetic electron bunches in the SMLWFA regime, which has been performed successfully for the first time in $2006 .{ }^{17,18}$ This quasimonoenergetic acceleration of electrons is the result of a combination of strong self-modulation and subsequent bubble acceleration.

\section{EXPERIMENTAL ENVIRONMENT}

In this section, the experimental setup and the laser and plasma parameters are described. We used the multiterawatt JETI laser with a pulse duration of $\tau_{L} \approx 80$ fs. To our knowledge, no longer laser pulse has yet been used successfully to produce quasimonoenergetic electron bunches. Together with a high peak plasma density of $n_{e} \approx 8 \times 10^{19} \mathrm{~cm}^{-3}$, the selfmodulation condition $\tau_{L}>\tau_{p} \approx 13$ fs was easily fulfilled. The laser pulse energy amounted to about $700 \mathrm{~mJ}$ on target and was focused to an intensity of $I \approx 5 \times 10^{19} \mathrm{~W} / \mathrm{cm}^{2}$, corresponding to a dimensionless field amplitude of $a_{0}$ $=e E /\left(m_{0} \omega_{L} c\right) \approx 5$, where $e$ and $m_{0}$ are the electron charge and mass, $E$ is the maximum electric field in the laser focus, $\omega_{L}$ is the laser light frequency, and $c$ is the speed of light.

Figure 1 shows a schematic of the experimental setup. The laser pulse comes from the left and is focused into a high density helium gas jet with a nearly Gaussian density profile, where $\tau_{L}>\tau_{p}$ is valid in the rising slope of the plasma from a density of $n_{e}>2 \times 10^{18} \mathrm{~cm}^{-3}$ on. Therefore, substantial self-modulation of the original laser pulse sets in and leads to much shorter laser pulse fragments after a propagation distance of a few 100 microns. Bubble acceleration can then be triggered by these pulse fragments, and quasimonoenergetic electron bunches are generated and can be detected after they have left the gas jet.

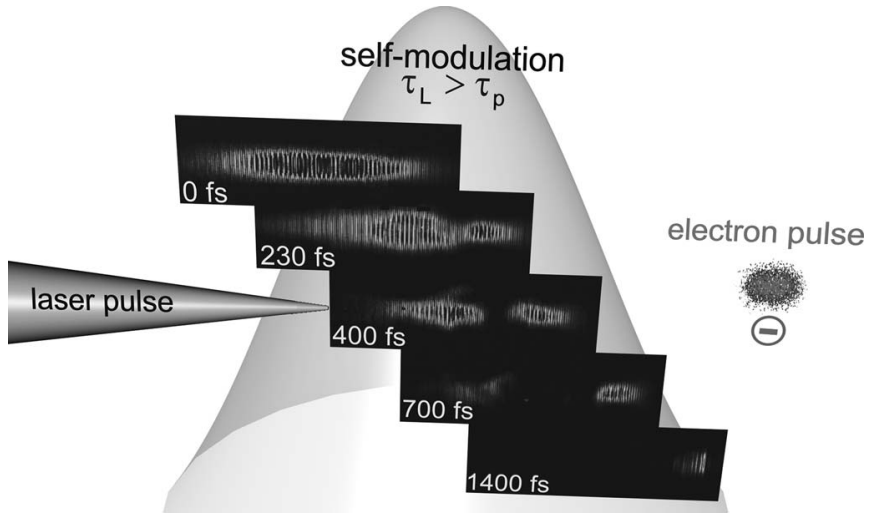

FIG. 1. Schematic of the experimental setup. The laser pulse with a duration of about 80 fs enters the high density helium gas jet, where self-modulation shapes the laser pulse into short fragments, which trigger quasimonoenergetic electron bubble acceleration.

In Fig. 2, the density profile of the nearly Gaussian gas jet with a peak density of about $n_{e} \approx 8 \times 10^{19} \mathrm{~cm}^{-3}$ and with a full width at half maximum (FWHM) of about $700 \mu \mathrm{m}$ as used in the experiment is plotted. In relation to the incident laser pulse with a duration of $80 \mathrm{fs}$ (or a length of $24 \mu \mathrm{m}$, respectively) the almost instantly ionized helium plasma initially has self-modulating density (i.e., where the plasma period $\tau_{p}<\tau_{L}$ ) on a distance of much more than $1 \mathrm{~mm}$. However, during the process of self-modulation in the rising slope of the plasma, the laser pulse fragments' lengths as well as intensities decrease. Therefore self-modulation in the falling slope behind the density maximum of the symmetric gas jet is weaker than in the rising slope, and the condition $\tau_{p}>\tau_{L}$ is reached closer to the density maximum in the falling slope than $\tau_{p}<\tau_{L}$ was reached in the rising slope. In this connection, it shall be noted here that although gas jet profiles are usually specified by plotting the plasma electron density $n_{e}$, it is the plasma wavelength $\lambda_{p}$ or the plasma frequency $\omega_{p}$ $=2 \pi c / \lambda_{p}$ which are the directly relevant plasma parameters for self-modulation as well as bubble formation. Since the plasma wavelength and period scale as $\lambda_{p} \propto \tau_{p} \propto n_{e}^{-1 / 2}$, pos-

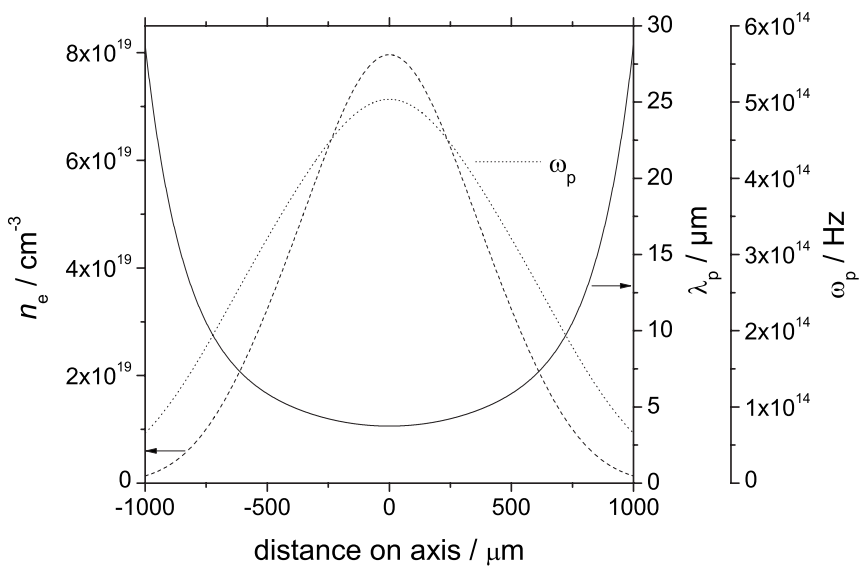

FIG. 2. Profile of gas jet density $n_{e}$, plasma frequency $\omega_{p}$, and wavelength $\lambda_{p}$, respectively. The directly important physical quantity for wakefield acceleration is the plasma wavelength $\lambda_{p}$, scaling as $n_{e}^{-1 / 2}$ and thus is leveling out partly the peaked density profile as well as possible local density inhomogeneities. 

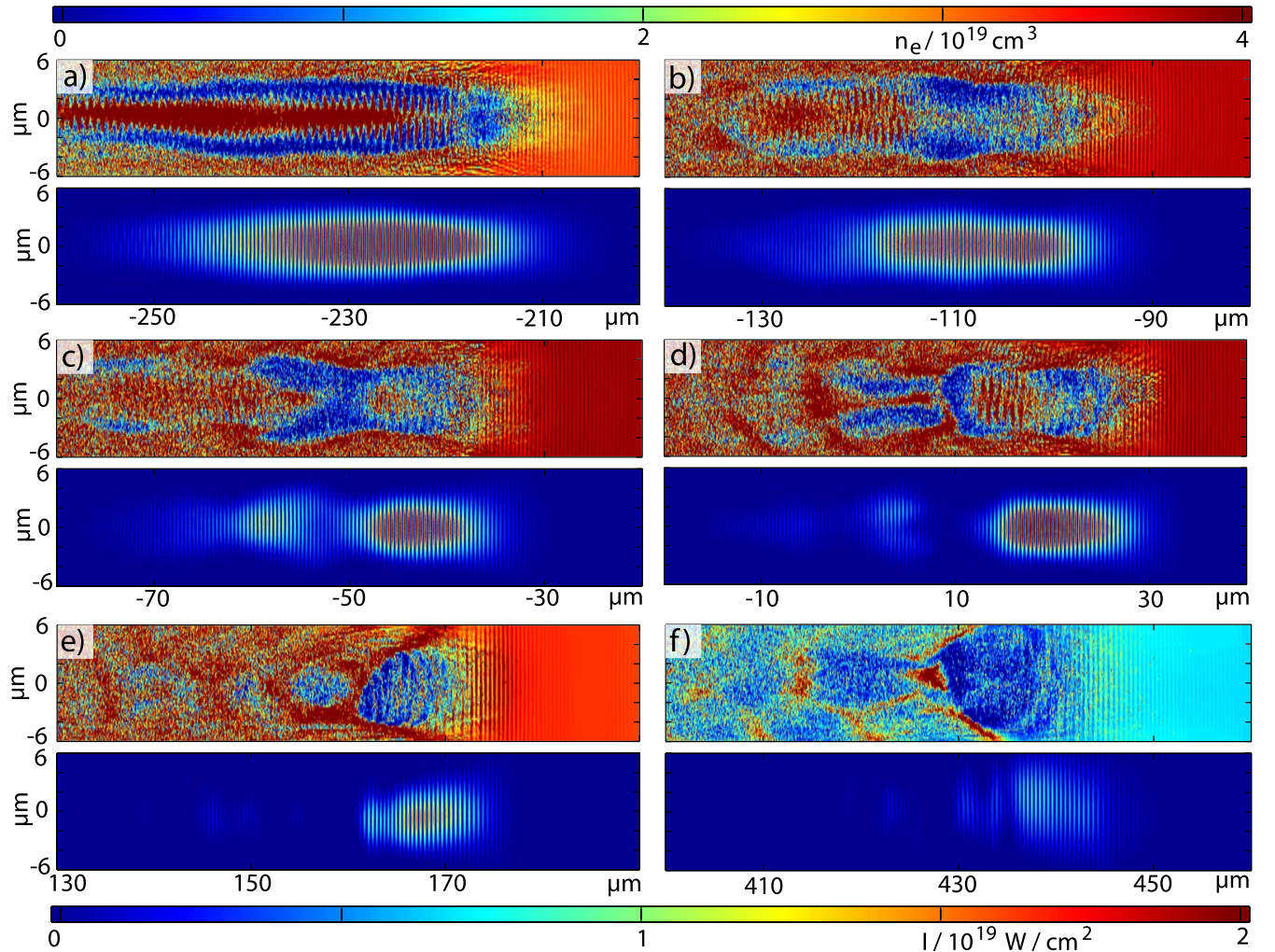

FIG. 3. (Color online) PIC simulation results with ILLUMINATION with a comparably low gas density of $n_{e} \approx 4 \times 10^{19} \mathrm{~cm}^{-3}$. Weak self-modulation of the laser pulse (bottom windows) leads too late to small enough pulse fragments to trigger electron bubble acceleration (top windows). Note that while the 3D simulation box had a size of $27 \times 27 \times 144 \mu \mathrm{m}^{3}$, much smaller windows are depicted here in order to save space and to focus on the middle of the simulation boxes where the main action takes place.

sible local density inhomogeneities have less impact and the physically relevant plasma frequency profile changes significantly slower than the density profile indicates.

Self-modulation is connected to Raman forward scattering (RFS) effects. ${ }^{19}$ Here, the scattered wave propagates in the same direction as the laser pulse, so that it acts back on the laser pulse as well as the plasma wave. For different regimes of RFS and during growth of the instability, different temporal growth rates $\Gamma$ occur. ${ }^{19}$ All growth rates increase strongly with laser intensity $a_{0}$ and plasma density $n_{e}$, which is the reason why self-modulation is promoted by high intensities and plasma densities.

\section{PARTICLE-IN-CELL SIMULATIONS}

Using such high intensities and gas densities is the key for reaching self-modulated quasimonoenergetic electron acceleration with comparably long laser pulses. This has been investigated in detail by performing three-dimensional (3D)particle-in-cell simulations (PIC) with the code ILLUMINATION at the Max-Planck-Institut für Quantenoptik in Garching. ${ }^{20}$ The discussion of these PIC simulation results presented in this section is connected to Sec. IV, where semianalytical scaling laws are put into context with the simulations and the experiments.

For the simulations, a moving window with a size of $27 \times 27 \times 144 \mu \mathrm{m}^{3}$ with $64 \times 10^{6}$ particles and $144 \times 144$ $\times 3080$ cells with one particle per cell was applied. The in- teraction with the nearly Gaussian gas jet with a profile such as in Fig. 2 has been simulated for peak densities of

- $n_{e} \approx 4 \times 10^{19} \mathrm{~cm}^{-3}$ ("low density"),

- $n_{e} \approx 8 \times 10^{19} \mathrm{~cm}^{-3}$ ("medium density," as in the experiment), and

- $n_{e} \approx 3.2 \times 10^{20} \mathrm{~cm}^{-3}$ ("high density").

Snapshots of the electron density (top windows) in the laser polarization plane and the laser pulse intensity (bottom windows) for the low (peak) density of $n_{e} \approx 4 \times 10^{19} \mathrm{~cm}^{-3}$ are shown in Fig. 3. The laser pulse propagates to the right and the longitudinal coordinate axis is set to zero at the maximum of the symmetric gas jet. Far from the gas jet center, in the low density part of the rising slope of the gas jet, self-modulation is weak and leads to significant fragmentation of the laser pulse not until close to the density maximum [in Fig. 3(c)]. Later, when the leading pulse fragment is finally small enough to fit into a plasma period [in Fig. 3(e)], for one thing the laser pulse is not intense enough anymore to effectively expel the electrons from the axis and trigger bubble acceleration due to energy depletion, and for another the plasma wavelength now increases relatively quickly in the falling slope of the gas jet which is additionally impedimental for bubble formation. The laser pulse afterwards propagates for more than $300 \mu \mathrm{m}$ through the gas jet until it is finally completely depleted.

In contrast, Fig. 4 illustrates the effects of (too) high plasma density. Here, self-modulation is so strong that there 

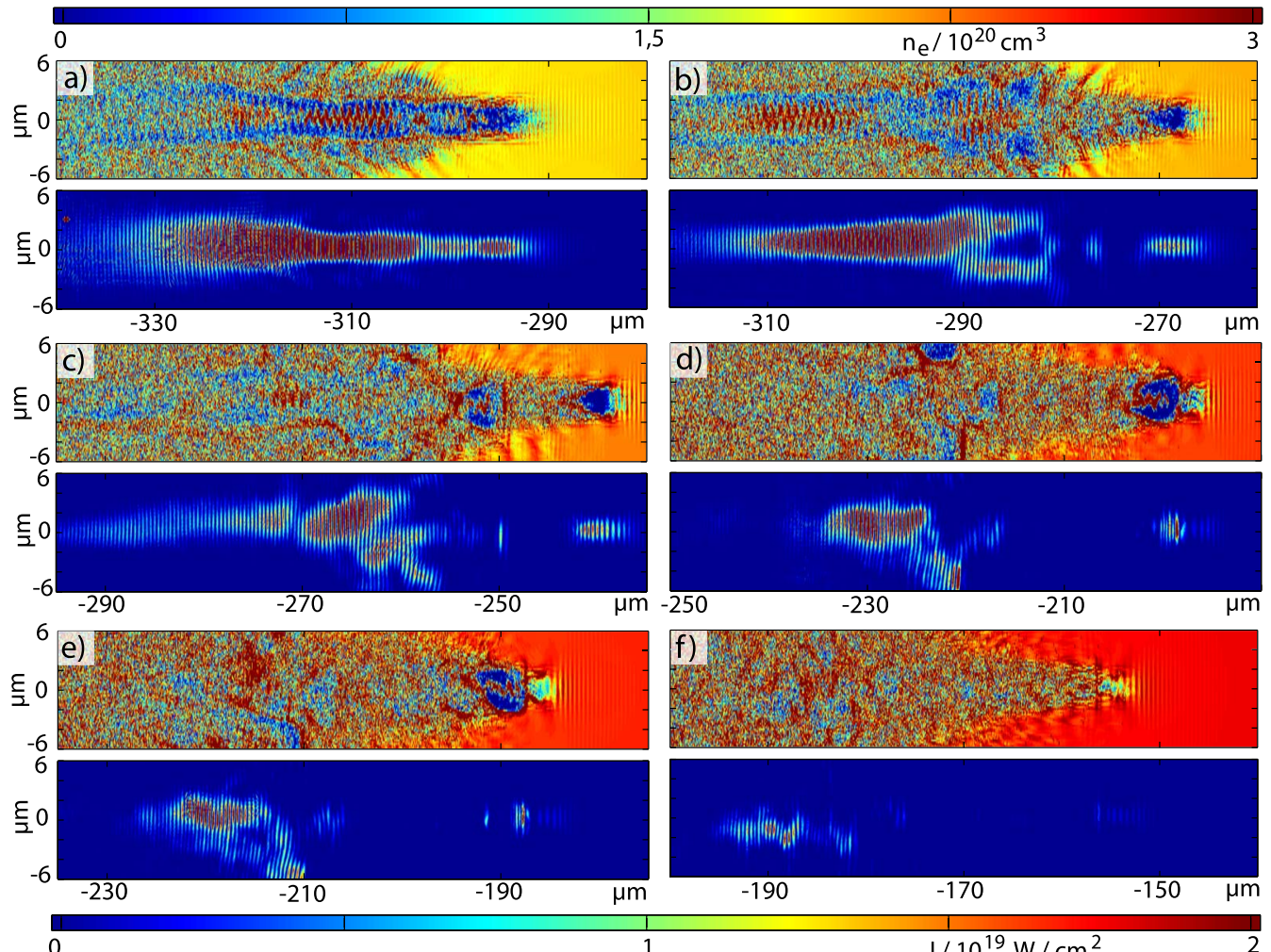

FIG. 4. (Color online) PIC simulation results with ILLUMinATION with an especially high electron density of $n_{e} \approx 3.2 \times 10^{20} \mathrm{~cm}^{-3}$. Due to the faster growth rates significant self-modulation of the laser pulse (lower pictures) starts very early and leads to longitudinal and transversal filamentation and the formation of one or even two bubbles [see (c) and Fig. 5 for a zoomed view of the same picture]. Laser depletion is reached before the beam has arrived at the plasma peak density.

is excessive self-modulation already in the beginning of the interaction. The laser pulse (front) shows fragmentation already $300 \mu \mathrm{m}$ in front of the density maximum [in Fig. 4(a)]. At the same time, self-focusing is drastically enhanced, and although the same laser pulse parameters have been used as for the run with the low gas density, the minimal laser pulse diameter is here approximately three times lower [e.g., compare Fig. 3(a) with Fig. 4(a)]. The rear part of the pulse is much less subject to self-focusing as well as selfmodulation, because this part of the laser pulse experiences a substantially disturbed plasma. Therefore, stochastic electron density and momenta distributions prevent instability growth here. However, the back of the pulse is relatively safe from the effects of the instabilities only in the beginning. At later times, when stronger fragmentation has developed and the distances between fragments grow larger, self-modulation as well as self-focusing passes on to the back of the pulse, too.

The pulse also filaments transversally. In Fig. 4(b), a laser pulse filament has formed which is bent away from the main pulse and decays on a distance of less than $100 \mu \mathrm{m}$ [see Figs. 4(c)-4(e)]. Although substantial pulse energy is lost for electron acceleration in the forward direction via such processes, some of the forming pulse fragments are short and powerful enough to trigger bubble acceleration. In Fig. 4(c), the first two pulse fragments are able to drive a bubble. This can be seen more clearly in Fig. 5(a), where a zoomed view of the two bubbles is depicted with a different color coding. Here, the second laser pulse fragment is sig- nificantly shorter than the first one. As a consequence, electrons are trapped only in the second bubble, where the driving self-modulated laser pulse fragment with a duration $\tilde{\tau}$ is much shorter than the plasma period $\tau_{p}$. However, after a distance of only $60 \mu \mathrm{m}$ [corresponding to Figs. 4(d) and 5 (b), respectively], the situation is completely different. The second laser pulse and the according bubble have completely vanished due to laser depletion and the chaotic plasma electron movement caused by the first bubble. Now, selfmodulation has shortened the first laser pulse fragment far

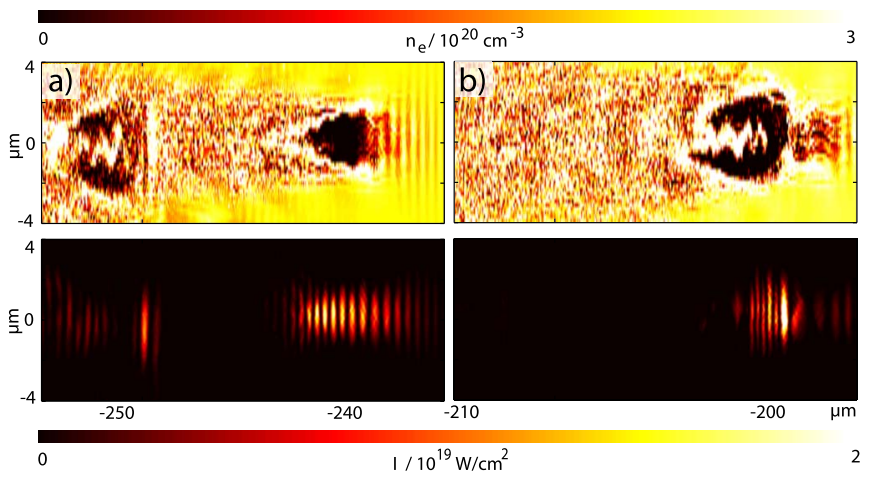

FIG. 5. (Color online) PIC simulation results with ILLUMINATION, depicting details from Figs. 4(c) and 4(d) with different color coding. From (a) it can be seen, that both pulse fragments are able to drive a bubble. Soon afterwards, the second bubble dies as a result of fading pulse fragment, while the leading pulse fragment is now short enough to trap and accelerate electrons. 


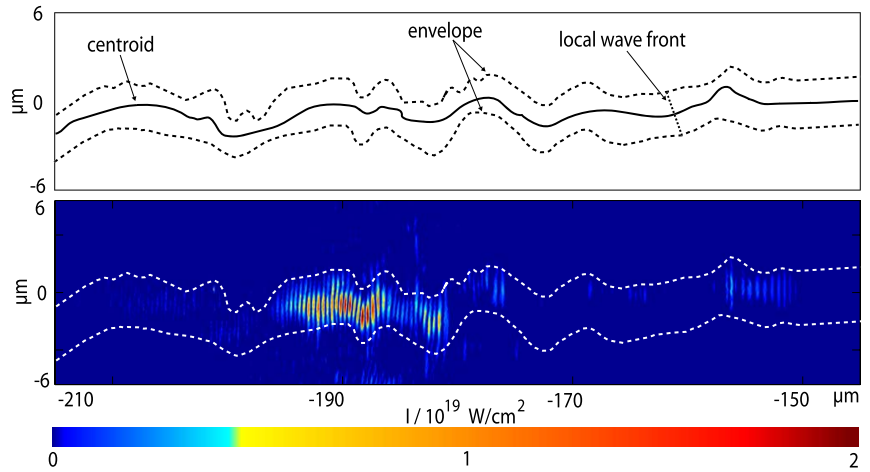

FIG. 6. (Color online) Snapshot of hosing of the laser pulse as observed with ILLUMination. Depicted is the laser pulse intensity as in Fig. 4(f) with more suitable color coding and with pulse envelope and centroid (top window).

enough to enable electron self-trapping and acceleration within the bubble.

This all happens far in front of the gas jet density maximum. The excessive self-modulation takes its toll: the laser pulse soon has too little energy to drive a bubble and depletes nearly completely on the next $100 \mu \mathrm{m}$.

Also, laser hosing can be observed well in the last three snapshots of Fig. 4. This hosing effect is visualized even better with a suitable color coding in Fig. 6. The bottom window shows the laser pulse intensity, with the two dotted white lines marking the pulse envelope. Note that this is a snapshot at one particular point of time and that the pulse envelope changes spatiotemporally during hosing. The top window depicts the envelope, the centroid (solid line) and indicates the tilting of the local wavefronts. Laser pulse hosing increases with rising density. ${ }^{21,22}$

The results of this high density simulation run therefore show extreme longitudinal self-modulation and fragmentation of the original laser pulse into a train of several laser pulse fragments, transversal self-focusing and filamentation as well as laser hosing. All of these instability effects increase with increasing density in the rising slope of the gas jet.

Next, simulations have been carried through for a medium gas peak density of $n_{e} \approx 8 \times 10^{19} \mathrm{~cm}^{-3}$, as used in the
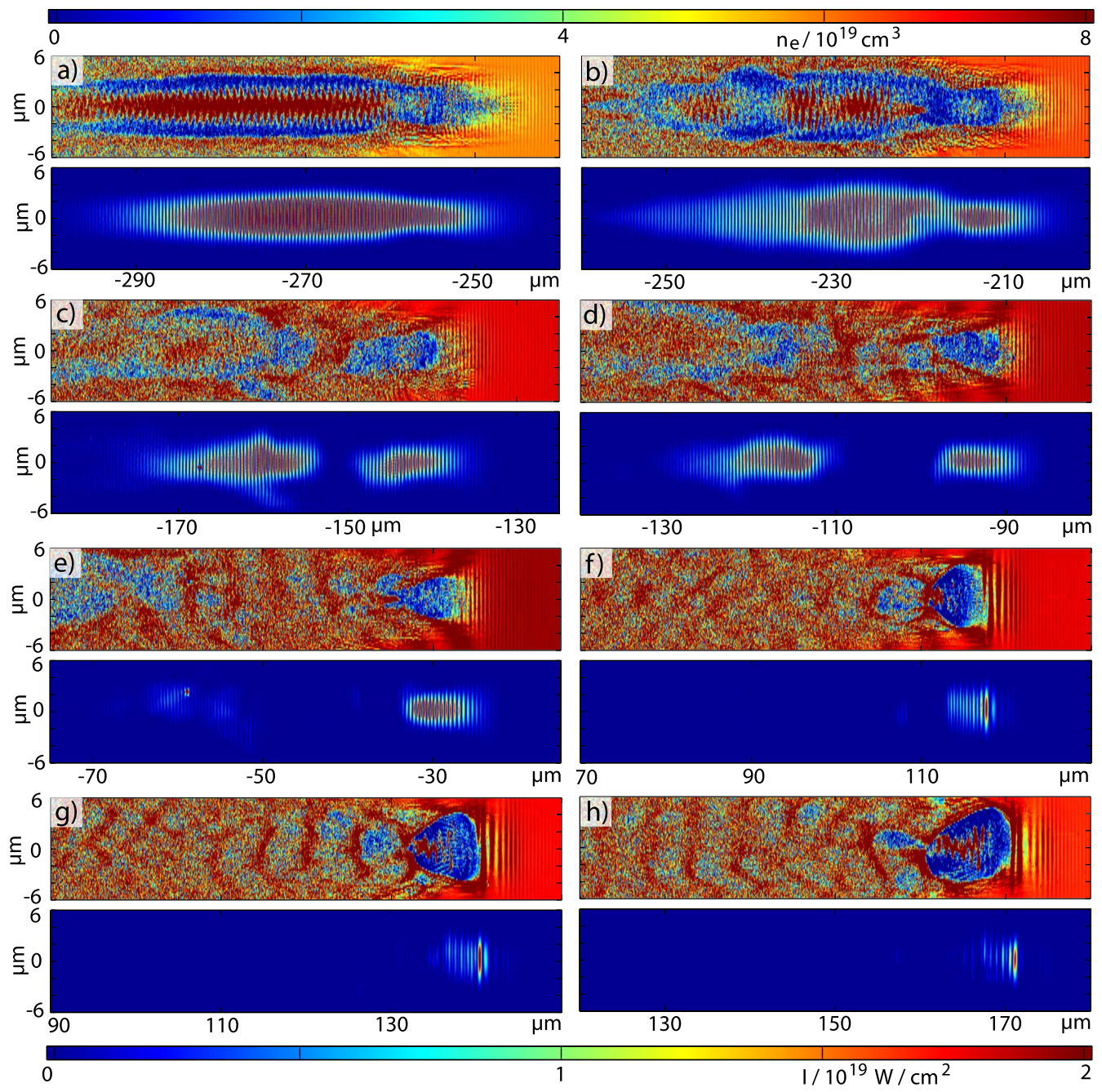

FIG. 7. (Color online) PIC simulations with ILLUMinATION. A medium electron density of $n_{e} \approx 8 \times 10^{19} \mathrm{~cm}^{-3}$ such as in the experiment makes sure that self-modulation, fragmentation, and hosing are neither too strong nor too weak. Distinct but energetic laser pulse fragments are formed which lead to the generation of a comparably stable bubble with a high electron density bunch accelerated inside. 
experiments. Figure 7 summarizes the results for this run. Here, self-modulation is moderate and leads to the formation of two pronounced, energetic laser pulse fragments [snapshots Figs. 7(b)-7(e)]. Only the leading laser pulse fragment survives the density peak, and is by then already short but at the same time still energetic enough to trigger a fully evolved bubble, in which electrons are trapped and accelerated in the falling slope of the gas jet [snapshots Figs. 7(f)-7(h)].

To sum up, the simulations show that the bubble acceleration regime can be reached after substantial selfmodulation even with laser pulses which are much too long initially. However, due to this dynamic process with constantly changing laser and plasma parameters, a large part of the laser energy is depleted and is not available for acceleration anymore, and various instabilities evolve. Nevertheless, in Sec. IV we will use estimations, results from the PIC simulations and experimental data in order to examine to what extent this self-modulated bubble regime can be described by frameworks which have been derived for the direct bubble regime, where the laser-plasma-parameters are sufficient to trigger a bubble right at the beginning of the interaction without any need for self-modulation or selfshortening of the driving laser pulse.

\section{COMPARISON WITH SCALING LAW PREDICTIONS}

Scaling laws which describe the electron acceleration process have been found and were published in Refs. 23-26. In Refs. 23-25, a similarity theory has been introduced and used to derive a power threshold $P_{\text {bubble }}^{\text {crit }}$ that the laser pulse with duration $\tau_{L}$ and power $P_{L}$ must overcome in order to trigger bubble acceleration. This power scales approximately as

$$
P_{L}>P_{\text {bubble }}^{\text {crit }} \approx\left(\frac{\tau[\mathrm{fs}]}{\lambda[\mu \mathrm{m}]}\right)^{2} \times 30 \mathrm{GW} .
$$

In our experiments as well as the simulations, the laser power of the incident $80 \mathrm{fs}$ laser pulse amounted to $\approx 8 \mathrm{TW}$, whereas following in Eq. (1), bubble acceleration would require $P_{L} \gtrsim 300 \mathrm{TW}$. This is in accordance with the simulations, where no bubble formation can be observed in the beginning of the interaction, where the laser pulse is still long and not self-modulated yet.

Furthermore, there are "geometrical" requirements, connected to the laser pulse shape and intensity (pulse duration $\tau$, focus diameter $w_{0}, a_{0}$ ) as well as to the plasma wavelength $\lambda_{p}$. This is intuitively clear, because the laser pulse must be intense enough to blow out the electrons from the axis effectively, and must fit into the approximately spherical bubble structure both transversally and longitudinally. It should also be self-guided over as long a distance as possible without changing its shape. The normalized bubble size $k_{p} R$ is determined mainly by the plasma wavelength $\lambda_{p}$ or the wave number $k_{p}=2 \pi / \lambda_{p}$, respectively.

By equating that the ponderomotive force the laser pulse exerts on the plasma electrons with the counteracting force of the ions which can be assumed to be stationary on this timescale, an optimal laser spot size of $k_{p} w_{0} \approx k_{p} R \approx \zeta \sqrt{a_{0}}$ can be matched to the (local) plasma density $\lambda_{p}{ }^{24,26}$ The $\zeta$-parameter ranges from $\zeta \approx 1-2$, depending on laser pulse polarization and whether periodic transversal self-focusing and defocusing shall be suppressed, for example. Generally, softer focusing leads to reduced laser pulse and bubble size oscillations.

The longitudinal laser pulse duration $\tau$ has to fit to the plasma density $n_{e}$, too. The longitudinal elongation of the laser pulse should not be bigger than the blowout or bubble radius, $c \tau \lesssim R$. Experimentally, the laser pulse duration is a parameter which imposes the most rigid constraint on the interaction. While an increase in duration can be realized relatively simply via various mechanisms, its minimum is fixed by the bandwidth of the pulse spectrum. One generally works with the shortest available pulse duration, and then sets the plasma electron density to a value which allows the highest obtainable wakefield strength by varying the gas density. For a Gaussian, linearly polarized laser pulse this is reached for $c \tau \approx \lambda_{p} / 2$. However, if the laser pulse is too long, the condition $c \tau \lesssim R$ implies that for a correspondingly larger $R$ the intensity $a_{0}$ which is required due to the condition $k_{p} R \approx \zeta \sqrt{a_{0}}$ would soon be too high to be realized. In such a case, self-modulation is an effective way to obtain matched interaction.

In the experiment, a maximum electron plasma density of $n_{e} \approx 8 \times 10^{19} \mathrm{~cm}^{-3}$, corresponding to a plasma wavelength of $\lambda_{p} \approx 3.7 \mu \mathrm{m}$, yielded the electron bunches with the highest energies. Under the simple assumption of homogeneous, sinusoidal self-modulation, the average FWHM durations of self-modulated pulse fragments can be estimated to $\tilde{\tau} \approx \lambda_{p} / 2 c \approx 6$ fs. The simulation results as depicted in Fig. 7 for the same plasma density demonstrate that such laser pulse fragments (and even shorter ones) can indeed be formed during the self-modulation process. The longitudinal requirement for matched interaction can be fulfilled for such pulse fragment durations for pulse radii down to $R \gtrsim c \widetilde{\tau}$ $\approx 1.8 \mu \mathrm{m}$.

With regard to the transversal condition $k_{p} R \approx \zeta \sqrt{a_{0}}$, a minimum linear plasma wavelength of $\lambda_{p} \approx 3.7 \mu \mathrm{m}$ (at the maximum density at gas jet center) and a minimum spot size of $R \approx 1.8 \mu \mathrm{m}$ (arising from the longitudinal requirement $R \gtrsim c \widetilde{\tau}$ ) require intensities ranging from $a_{0} \approx\left[2 \pi R /\left(\lambda_{p} \zeta\right)\right]^{2}$ $\approx 2.3-9.3$, depending on the value of $\zeta$.

In Eq. (1) shows, that the formed pulse fragments' power $\widetilde{P}_{\text {bubble }}^{\text {crit }}$ necessary for triggering bubble acceleration scales with the squared pulse fragments' duration $\tilde{\tau}^{2}$, and correspondingly the required energy of the pulse fragment as $\widetilde{\tau}^{3}$. Therefore, the radical reduction in the laser pulse duration due to self-modulation by more than an order of magnitude is very favorable. In addition, the laser pulse fragments' power can even increase during the self-modulation process. This results from longitudinal energy bunching and can also be seen in simulations. Assuming a laser pulse fragment energy of $\widetilde{E} \approx 50 \mathrm{~mJ}$ and a fragment duration of $\tilde{\tau} \approx 6 \mathrm{fs}$, the original laser pulse power of $P \approx \widetilde{P} \approx 8 \mathrm{TW}$ is maintained. For such parameters, in Eq. (1) amounts to $\widetilde{P}_{\text {bubble }}^{\text {crit }}$ $>1.7 \mathrm{TW}$ and thus can be easily fulfilled. Pulse fragments with powers above these values can therefore trigger bubble acceleration. As an upper limit, one can assume that the front 

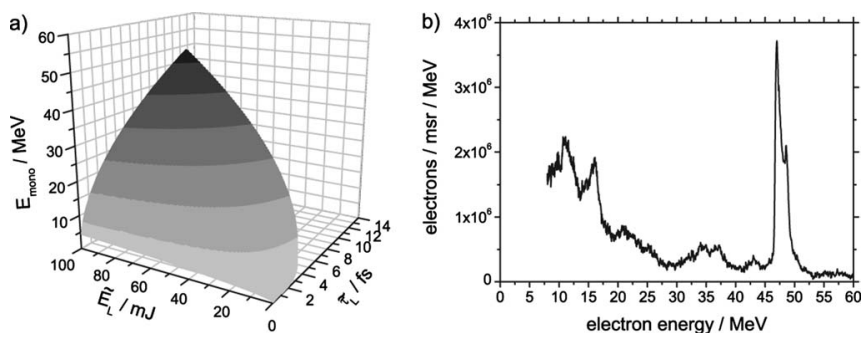

FIG. 8. (a) Scaling law prediction from Refs. 24 and 25 for the peak quasimonoenergetic electron energy, and (b) experimentally measured, quasimonoenergetic spectrum.

of the laser pulse with an energy of $700 \mathrm{~mJ}$ in $80 \mathrm{fs}$ is cut off via self-modulation in the gas jet density maximum with a plasma period $\tau_{p}=\lambda_{p} / c \approx 12$ fs to form a compressed pulse fragment with an energy of $\widetilde{E} \approx 100 \mathrm{~mJ}$ in $6 \mathrm{fs}$ FWHM, corresponding to a power of $\widetilde{P} \approx 15 \mathrm{TW}$.

After showing that via self-modulation the bubble regime can be entered, we will now have a closer look on the quantitative predictions of the semianalytical scaling laws, and will compare those to our experimental results. It shall be stressed again that these scaling laws have been derived for cases where self-modulation does neither play a major role nor is necessary to reach the stage where self-injection and quasimonoenergetic electron acceleration is possible. This said, we will act as if a fresh laser pulse with parameters as estimated above after self-modulation (energies up to $\widetilde{E}$ $\approx 100 \mathrm{~mJ}$ and pulse durations down to $\tilde{\tau} \approx 6 \mathrm{fs}$ ) would be incident on the plasma.

From the similarity theory, a prediction for the maximum energy of the quasimonoenergetic peak $E_{\text {mono }}$ in the electron spectrum has been obtained, ${ }^{24,25}$ which can be written as

$$
E_{\text {mono }} \approx 0.1 \mathrm{MeV} \sqrt{\frac{P}{P_{\text {rel }}}} \frac{\tau[\mathrm{fs}]}{\lambda_{L}[\mu \mathrm{m}]} .
$$

By using the laser pulse fragments' power $P=\widetilde{P}=\widetilde{E}_{L} / \widetilde{\tau}$ one can plot the maximum quasimonoenergetic bunch energy in dependence of laser pulse energy $\widetilde{E}_{L}$ and duration $\tilde{\tau}$, scaling as $\widetilde{E}_{L} \propto \widetilde{\tau}^{3}$. This has been done in Fig. 8(a). Only those pairs of $\widetilde{E}_{L}$ and $\widetilde{\tau}$ have been plotted which satisfy the power threshold condition $P_{\text {bubble }}^{\text {crit }} \approx(\tau[\mathrm{fs}] / \lambda[\mu \mathrm{m}])^{2} \times 30 \mathrm{GW}$ [Eq. (1)].

Experimentally, the electron bunches with the highest energy reached up to $50 \mathrm{MeV}$. An according measured spectrum is given in Fig. 8(b).

Applying the scaling law prediction from the similarity theory [Eq. (2)], an electron bunch energy of $50 \mathrm{MeV}$ can be reached by bubble-driving laser pulse fragments with an energy of $\widetilde{E}_{L} \approx 100 \mathrm{~mJ}$ and a duration of $\tilde{\tau} \approx 12 \mathrm{fs}$. As shown above, such pulse energy and duration parameters occur during self-modulation, rendering experiment, scaling law, and simulations consistent.

A different energy scaling was obtained in Ref. 26 and is given as

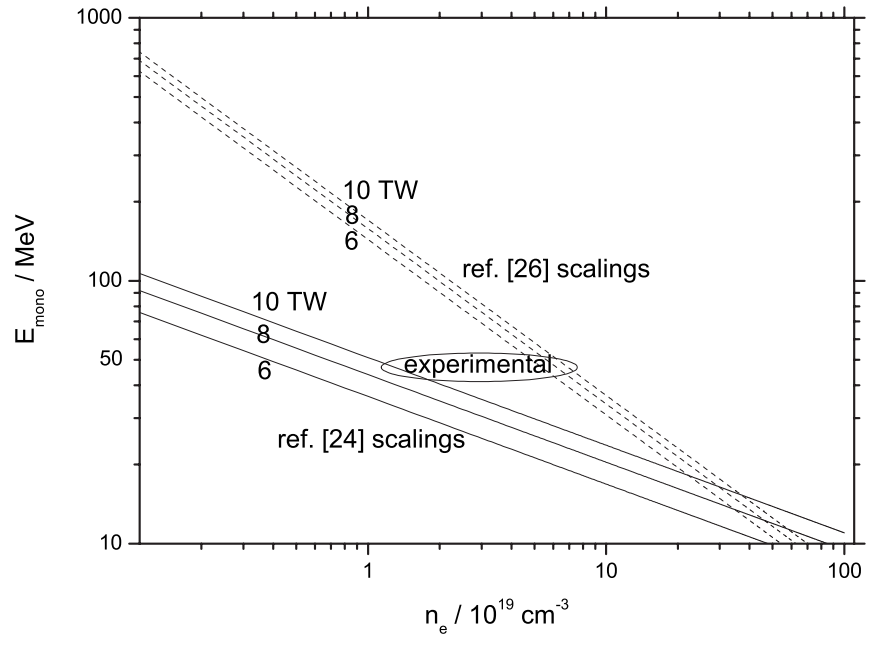

FIG. 9. Comparison of scaling law prediction for the maximum electron energies from Eqs. (4) and (3) with experimentally observed values.

$$
E_{\text {mono }}[\mathrm{GeV}] \approx 3.8\left(\frac{P}{P_{\mathrm{rsf}}^{\text {crit }}}\right)^{-2 / 3} \frac{P[\mathrm{TW}]}{100},
$$

where $P_{\mathrm{rsf}}^{\text {crit }}$ is the threshold for relativistic self-focusing and amounts to $P_{\mathrm{rsf}}^{\text {crit }}=\left(2 m^{2} c^{5} / e^{2}\right)\left(\omega_{0}^{2} / \omega_{p}^{2}\right) \approx 17\left(\omega_{L}^{2} / \omega_{p}^{2}\right)[\mathrm{GW}]$ $=17\left(n_{c} / n_{e}\right)[\mathrm{GW}]$.

The difference between both results [Eqs. (2) and (3)] is extensively discussed in Ref. 26, and it is argued that this energy scaling is valid for normalized laser amplitudes down to $a_{0} \approx 2$. In contrast, the pioneering scaling from Refs. $23-25$ is strictly valid for very high intensities of $a_{0} \gtrsim 20$. In order to compare the experimental results with both scalings, Ref. 26 rewrites Eq. (2) by employing the expression for the optimal spot size $k_{p} R \simeq k_{p} w_{0} \simeq \sqrt{a_{0}}$ from Refs. 23-25. Using the natural relativistic power unit $P_{\text {rel }}=4 \pi \epsilon_{0}\left(m^{2} c^{5} / e^{2}\right)$ $\approx 8.5 \mathrm{GW},{ }^{23-25}$ the density dependence is revealed in the form $^{26}$

$$
E_{\text {mono }}=0.16 m c^{2} \frac{c \tau}{w_{0}}\left(\frac{P}{P_{\text {rel }}}\right)^{2 / 3}\left(\frac{n_{c}}{n_{e}}\right)^{1 / 3}
$$

or as an engineering formula for $\lambda=800 \mathrm{~nm}$

$$
E_{\mathrm{mono}} \approx 24 \mathrm{MeV} \times \frac{c \tau}{w_{0}} \frac{P[\mathrm{TW}]^{2 / 3}}{n_{e}\left[10^{18} \mathrm{~cm}^{-3}\right]^{1 / 3}} .
$$

The maximum energies of the quasimonoenergetic electron bunches predicted by Eq. (4) on the one hand and by Eq. (3) on the other hand are plotted in Fig. 9 versus the plasma electron density for laser pulse fragment powers of 6,8 , and 10 TW. In doing so, an approximately round beam with $\left(c \tau / w_{0} \approx R\right) \approx 1$ has been assumed with Eq. (4), while cigarlike $\left(c \tau>w_{0}=R\right)$ and pancakelike beams $\left(c \tau<w_{0} \approx R\right)$ would yield different energies.

The plots mirror the stronger density dependence of Eq. (3) when compared to Eq. (4). The maximum experimentally observed electron energies are indicated by the ellipsoid in Fig. 9. The ellipsoid ranges from densities of $n_{e} \approx 1$ $\times 10^{19} \mathrm{~cm}^{-3}$ to the maximum experimental density of $n_{e}$ $\approx 8 \times 10^{19} \mathrm{~cm}^{-3}$, reflecting the fact that bubble formation in the simulations is not always only observed at the maximum 

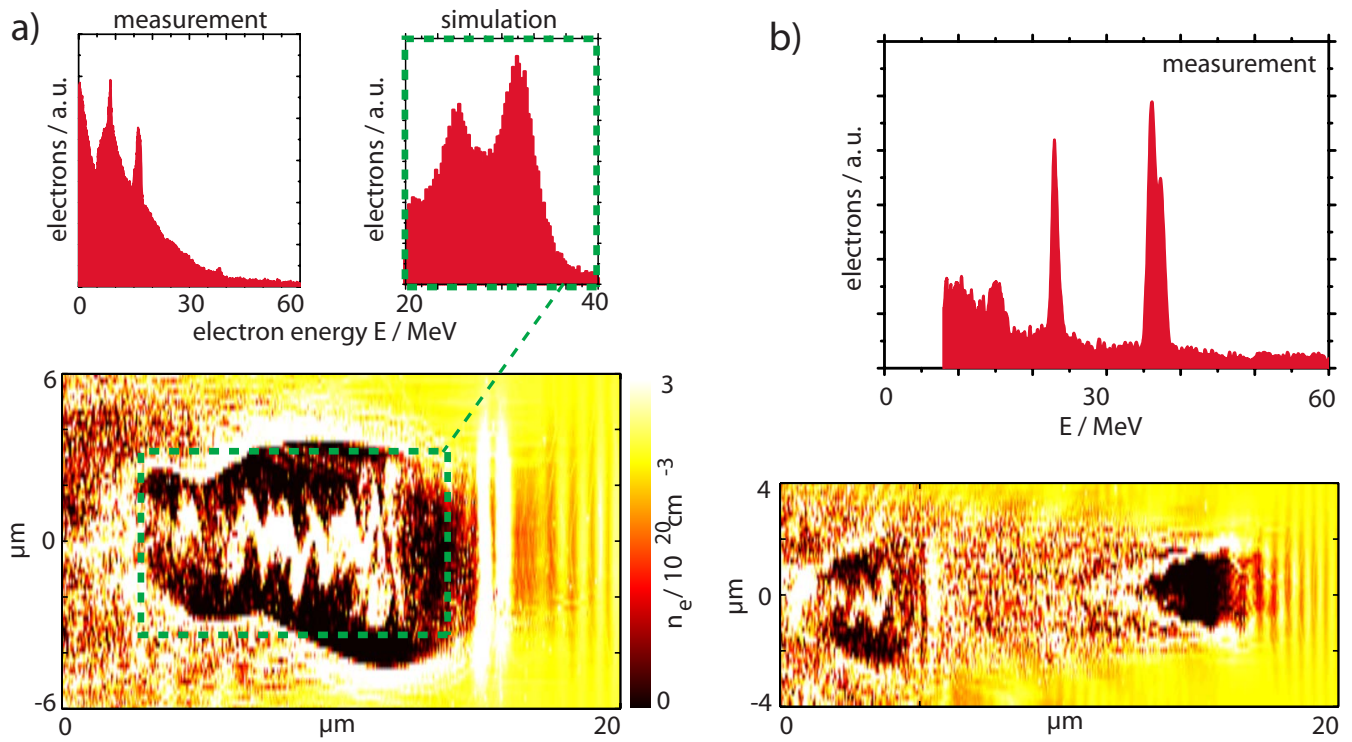

FIG. 10. (Color online) Two different scenarios of generation of multiply spiked electron spectra. (a) Generation of mildly spiked spectra due to the partial decay of an electron-accelerating plasma bubble. Left top corner: measured spectrum, right: spectrum of the electrons inside the green dashed obtained from simulation in the bottom snapshot, which depicts the according plasma density distribution in the simulation. (b) Pronounced electron double peaks can be generated when two laser pulse fragments drive bubbles. Top window: measured spectrum, bottom window: situation as observed in simulations.

density but sometimes already at lower values in the rising slope. Although the power of the self-modulated, driving laser pulse fragments which lead to the highest energies in the experiments is not exactly known and has to be estimated, the experimental values are not too far away from the scaling laws, indicating that the scaling laws do approximate the true values quite accurately even for the described complicated experimental setup which involves strong self-modulation.

Both scaling law frameworks do also allow for the prediction of the maximum number of quasimonoenergetic electrons in the created bunches. However, the charge of the quasimonoenergetic electrons measured in our experiments was lower by approximately two orders of magnitude when compared to both scaling law predictions for the maximum possible charge. In this context, it shall be noted that generally often a much higher charge is seen in PIC simulations when compared to the charge which can be confirmed by the experiments, which is still an open question and probably in part related to bunch extraction at the end of the gas jet. This comes in addition to the fact that in our experiments a more complex situation is present than in the simulations which quantified the scaling laws.

\section{ULTRASHORT ELECTRON DOUBLE BUNCHES}

In the experiments, sometimes multiple spikes in the electron spectra were observed. Different possible scenarios are identified to explain this feature, each with an entirely different set of characteristics.

In the one case, the spikes are not very pronounced. Such a situation corresponds to Fig. 10(a). Spectra such as the one depicted in the upper left corner of the figure have been measured during our experimental campaigns and show only moderately pronounced peaks at energies not higher than about $25 \mathrm{MeV}$. Such spectra can be reproduced by PIC simulations and a simulated example spectrum is given next to the measured one in Fig. 10(a). The corresponding electron density snapshot from the simulation is depicted at the bottom of the picture. For the energy spectrum, only electrons in the green dashed box with energies between 20 and $40 \mathrm{MeV}$ and which are moving in the forward direction have been counted. At this point of the simulation, the density snapshot shows furthermore that the shape of the electron cavitation is considerably different from the smooth round shape usually observed, which might be connected to the formation of the moderately spiked electron spectra.

The second scenario deals with completely different physical processes and is characterized by extremely pronounced double spikes, in contrast. The upper part of Fig. 10(b) shows a measured spectrum with two distinct peaks, the more energetic of which reaches approximately $35 \mathrm{MeV}$. It has been supposed already in Ref. 17 that such a double bunch can be created if self-modulation leads to the generation of two consecutive laser pulse fragments which can be separated by only few tens of femtoseconds because they are created from a laser pulse which is only $80 \mathrm{fs}$ long. If these laser pulses are both strong enough and meet the geometrical and gas density requirements discussed above, they can each drive a bubble and accelerate electrons to quasimonoenergetic energies. This can result in electron bunches which are therefore also separated by only few tens of femtoseconds. In the lower part of Fig. 10(b), the electron density window from the simulation run for the high density which has been shown before in Fig. 5 is given again in order to show the fundamental difference to the situation in Fig. 10(a).

The acceleration of electrons in two consecutive wave buckets and a temporal distance of the order of the plasma wavelength has later been shown experimentally with a different laser system by another group making use of transition radiation. ${ }^{27,28}$ However, this can also be attributed to a situation where the laser pulse does not extend over two plasma 

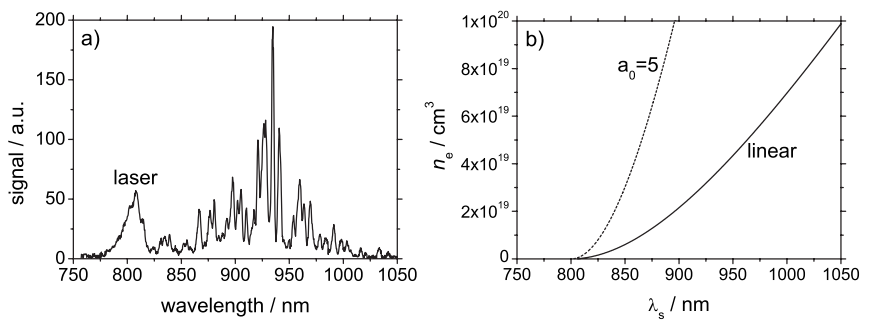

FIG. 11. Transmitted laser light spectrum. (a) In addition to the peak at the central laser wavelength at $800 \mathrm{~nm}$ there is a broad and strong Stokesshifted light portion. (b) Expected frequency of the Stokes peak in dependence on the local linear (solid line) and nonlinear plasma frequency (assuming $a_{0}=5$ ).

periods but nevertheless a second wave bucket is formed behind the laser pulse and the first bubble which is strong enough to accelerate electrons to quasimonoenergetic energies, as observed in PIC simulations. ${ }^{26,29}$ In Ref. 18, the formation of multiple spikes in the electron spectra has been ascribed to self-modulation, too.

\section{ADDITIONAL OBSERVATIONS IN EXPERIMENTS AND SIMULATIONS}

A strong experimental confirmation of RFS and pronounced self-modulation can be found in the transmitted light spectra, which we have measured with an optical spectrometer in the forward direction on axis. Figure 11(a) shows an example of a transmitted light spectrum which exhibits a strong peak close to the central laser wavelength $\lambda$ $\approx 800 \mathrm{~nm}$, but also several Stokes-shifted peaks at considerably higher wavelengths. For an experimental maximum electron density of $n_{e} \approx 8 \times 10^{19} \mathrm{~cm}^{-3}$ one would expect an upper bound at $\omega_{s}=\omega_{L}-\omega_{p}$ corresponding to $\lambda_{s} \approx 1020 \mathrm{~nm}$, which is confirmed by the spectrum. The maximum in the spectrum is at $930 \mathrm{~nm}$. This could be due to the fact that most self-modulation happens in the rising slope of the gas jet, where the plasma density is lower, and the laser intensity is higher. Additionally, the effective nonlinear plasma wavelength $\lambda_{p}^{\mathrm{NL}} \approx \lambda_{p} \sqrt{a_{0}}$ can be significantly higher than the linear one, which also leads to a shift toward lower wavelengths. Figure 11(b) shows the theoretical Stokes-shifted wavelength in dependence of the plasma density for the linear case $\left(a_{0}\right.$ $\lesssim 1$, solid line) and the nonlinear case $\left(a_{0}=5\right.$, dashed line), respectively. The peak in the spectrum at $930 \mathrm{~nm}$ therefore corresponds to an average plasma density of about $n_{e} \approx 3$ $\times 10^{19} \mathrm{~cm}^{-3}$ in case of the linear plasma wavelength.

An increase in the electron bunch emittance in the laser polarization plane has been observed in Ref. 30 and has been explained in terms of betatron oscillations driven by the laser field via PIC simulations and an analytical model in Ref. 31. The oscillation and microbunching of the electron bunch inside the bubble is clearly observable in our simulations, too. In Fig. 12(a) the top window shows a formed bubble in the plane of the polarization direction of the laser pulse, while the bottom window shows the corresponding transverse perpendicular direction taken from the 3D simulation. While there are strong oscillations of the electrons in the polarization direction, there is little to no such oscillation in the
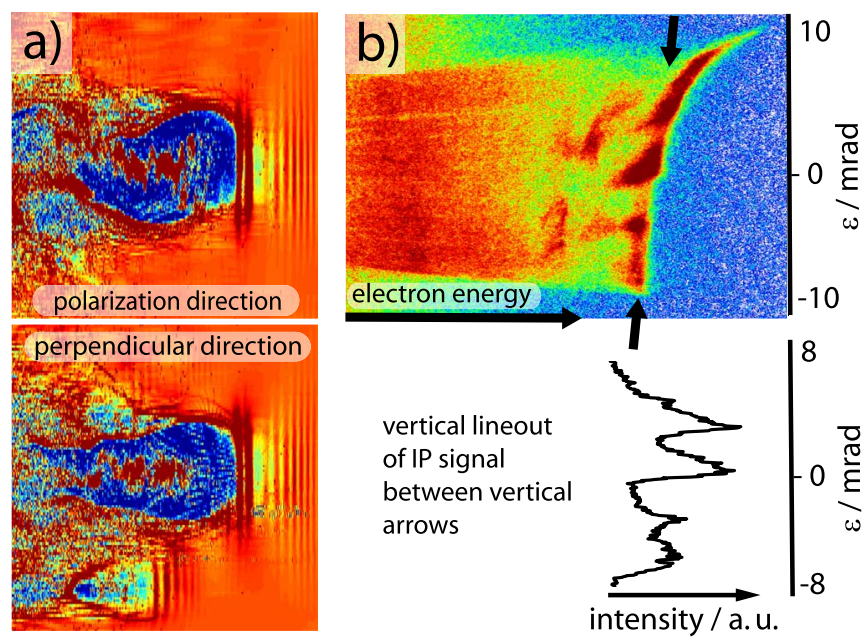

FIG. 12. (Color online) (a) PIC simulations [ILlumination (Ref. 20)] show oscillations of the electron bunch inside the bubble in the polarization direction of the laser field (top window) while there are considerably less oscillations in the perpendicular transverse direction (bottom window). (b) Experimental electron signal as detected on an image plate in the polarization direction showing a strong transversal fine structure.

perpendicular direction. The small fraction of the laser field which is still present inside the bubble can be responsible for this oscillation, as can be seen from the simulations in Fig. 7, for example.

In the experiments, the accelerated electrons were energy resolved in a spectrometer based on permanent magnets and detected by image plates. ${ }^{32}$ The detection plane was chosen in the direction of the laser polarization, thus allowing for observation of eventual beam inhomogeneities in this direction. Indeed, we sometimes observed a pronounced transversal fine structure in the spots the electrons created on the image plates. An example of such a strong transversal fine structure in a quasimonoenergetic signal is depicted in Fig. 12(b). The top window shows the color coded raw data of the electrons on the image plate, and the bottom window shows a lineout drawn through the signal at the position of the opposing (nearly) vertical arrows. The left hand side on the image plate corresponds to lower energy electrons and the right hand side to high-energy electrons. We attribute the lateral fine structure to the electrons oscillating in the laser polarization direction, at the end of the interaction leaving the gas jet into slightly different directions due to their different transverse positions and momenta.

The beam divergence $\Theta$ was measured in our experiments by means of a fluorescent beam viewer and amounted to values of $\Theta<10 \mathrm{mrad}$, which is comparable to other instances of quasimonoenergetic laser-plasma acceleration. We did not observe significant broadening in the direction of laser polarization on this beam viewer as in Ref. 30, but this effect may have been undetectable on the beam viewer due to a rather short distance from the interaction region $(12 \mathrm{~cm})$. As concerns beam emittance, the high plasma density and thus the small bubble radius leads to an especially small beam source size [scaling as $\left.\approx 1 / \sqrt{n_{e}}(\operatorname{Ref} .26)\right]$. For a beam size $b$ of about $b<5 \mu \mathrm{m}$ (as observed in simulations), an emittance of $\epsilon \lesssim \Theta b \approx 0.05 \mathrm{~mm}$ mrad can be estimated. For 
the observed peak electron energies of $\approx 50 \mathrm{MeV}$, this leads to a normalized emittance of $\epsilon_{n}=\gamma \beta \epsilon \lesssim 5 \mathrm{~mm} \mathrm{mrad}$, which is comparable to the parameters of beams generated in rftype accelerators. The small bubble size due to the high plasma electron densities causes not only a small transversal bunch size (and thus, small emittances) but also small longitudinal dimensions of the electron bunches. The electron bunch durations can be estimated to be of the order of those of the driving laser pulse fragments of down to $5 \mathrm{fs}$, only. Both effects, the ultrashort transversal and longitudinal dimension, lead to high initial electron densities or brightness of the bunches, despite the comparably rather low charge measured. This is a special feature of electron bunches generated in the SMLWFA regime. For example, the $50 \mathrm{MeV}$ peak could be additionally characterized by a brightness $B$ $=\left(I /(\gamma \beta \epsilon)^{2}\right)=\left(I / \epsilon_{n}^{2}\right) \approx 2.5 \mathrm{~A} \mathrm{~mm}^{-2} \mathrm{mrad}^{-2}$, where $I$ is the average current of the 5 fs electron bunch.

\section{CONCLUSION}

Quasimonoenergetic electron bunches can be generated in the SMLWFA regime with laser pulses of rather modest pulse powers. This is in contrast to theoretical predictions, which ruled out quasimonoenergetic electron acceleration for laser pulses such as those we have used in our experiments. These two seemingly contradictory results are resolved by the decay of the original laser pulse into several laser pulse fragments via self-modulation. If electron density and laser intensity are high enough, these clearly separated fragments can possess parameters sufficient to generate quasimonoenergetic electron bunches resulting from their interaction with the plasma. This is reproduced by PIC simulations, and the observed energies of the self-modulated electron bunches are in accordance with scaling law predictions, if one uses not the incident laser pulse parameters, but instead the parameters of the self-modulated laser pulse fragments.

High plasma electron density is the key parameter in SMLWFA. Longitudinal self-modulation is strongly connected to various other effects, such as transversal filamentation, laser hosing and photon deceleration, which are observed in simulations and are consistent with experimental data. These instabilities all get stronger when the plasma electron density is higher, which is why so far the quasimonoenergetic electron bunch production in this regime could not be stabilized as far as in matched LWFA.

On the other hand, the high plasma density leads to especially high accelerating fields in (quasimonoenergetic) SMLWFA. Furthermore, because $P_{\text {rsf }}^{\text {crit }}$ is reduced, a stronger self-focusing can lead to higher intensities, which, in turn, increases the accelerating field even more. Figure 13 gives a rough schematic overview on the accelerating fields of SMLWFA in comparison to LWFA, PWFA, and conventional rf cavity based accelerators, such as those at the Stanford Linear Accelerator Center (SLAC), or those produced for the TeV Energy Superconducting Linear Accelerator (TESLA) study and those discussed in the Compact Linear Collider (CLIC) study. Only the direct use of the (transverse) electric fields in focused laser pulses offers even higher accelerating

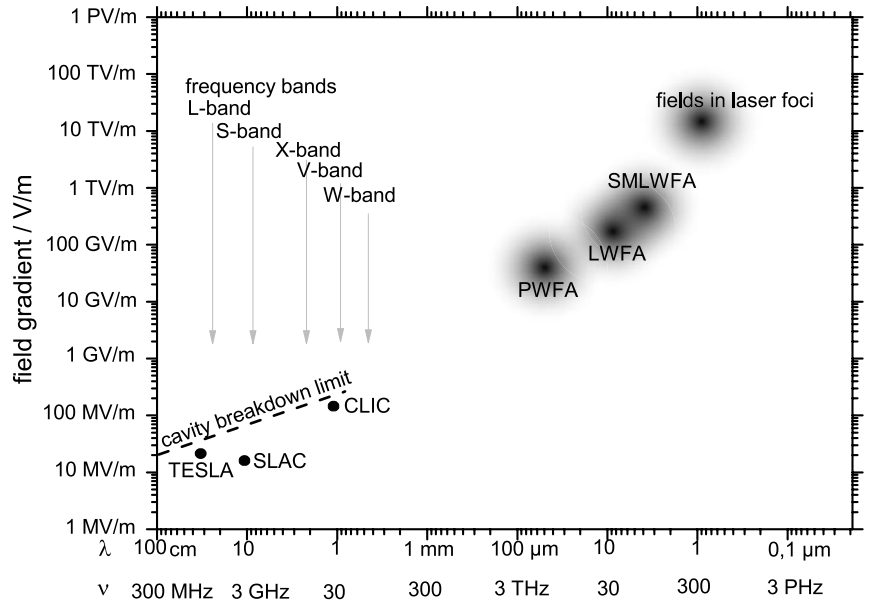

FIG. 13. Schematic overview on the accelerating fields of SMLWFA in comparison to other types of accelerators. Due to the high electron densities with SMLWFA, the (longitudinal) accelerating fields can be considerably higher than with LWFA or PWFA, and by many orders of magnitude higher than in conventional rf cavity based accelerators. Only the (transverse) electric fields in focused laser pulses can be even higher.

forces than with SMLWFA, but harvesting those is difficult due to the Lawson-Woodward theorem. . $^{33,34}$

As concerns electron energy, the high electron density has at the same time advantageous and disadvantageous effects. The dephasing distance is shorter due to a slower laser pulse group velocity, which is good because dephasing is necessary to some extent in order to generate a quasimonoenergetic electron spectrum. ${ }^{26}$ In contrast, early dephasing limits the overall energy gain, because the effective acceleration distance is reduced, which can cancel out the advantage of higher accelerating fields.

The extremely small accelerating structures in SMLWFA have profound effects on the emittance, pointing and brightness of the bunches, too. While initial emittance and brightness can be good due to the short transversal and longitudinal dimensions of the bunches, pointing is rather unstable because after all, the acceleration process is based on highly nonlinear interaction and the connected instabilities. It shall also be noted that due to the shorter dephasing length the thus limited energy gain leads to a heavier impact of the Coulomb forces which tend to drive apart the electron bunch, due to a smaller $\gamma$-factor of the electrons.

Another interesting feature of quasimonoenergetic SMLWFA is the possibility of formation of two pronounced quasimonoenergetic electron bunches by subsequent selfmodulated laser pulse fragments. Here, each laser fragment drives a bubble and accelerates electrons to relativistic, quasimonoenergetic energies. Future analyses should be carried out on this phenomena of multiple, consecutive, and intense electron bunches, because such systems with ultrashort distances between the bunches could be a wellsuited test bed to study electron bunch driver/witness scenarios which are seen as a possible route toward substantial energy enhancement, for example, via plasma wakefield accelerator stages as boosters for conventional accelerators. 


\section{ACKNOWLEDGMENTS}

This work was supported by DFG-Projects Transregio TR18 and Graduiertenkolleg GRK1203. We thank the staff at the Institut für Optik und Quantenelektronik at the FriedrichSchiller-Universität Jena, Germany, for reliable laser operation and excellent support during the experimental campaigns.

${ }^{1}$ V. Veksler, in Proceedings of CERN Symposium on High Energy Accelerators and Pion Physics (CERN, Geneva, 1956), Vol. 1, p. 80.

${ }^{2}$ G. I. Budker, in Proceedings of CERN Symposium on High Energy Accelerators and Pion Physics (CERN, Geneva, 1956), Vol. 1, p. 68.

${ }^{3}$ B. Fainberg, in Proceedings of CERN Symposium on High Energy Accelerators and Pion Physics (CERN, Geneva, 1956), Vol. 1, p. 84.

${ }^{4}$ A. I. Akhiezer and R. V. Polovin, Sov. Phys. JETP 3, 696 (1956).

${ }^{5}$ J. M. Dawson, Phys. Rev. 113, 383 (1959).

${ }^{6}$ T. Tajima and J. M. Dawson, Phys. Rev. Lett. 43, 267 (1979).

${ }^{7}$ S. P. D. Mangles, C. D. Murphy, Z. Najmudin, A. G. R. Thomas, J. L. Collier, A. E. Dangor, E. J. Divall, P. S. Foster, J. G. Gallacher, C. J. Hooker, D. A. Jaroszynski, A. J. Langley, W. B. Mori, P. A. Norreys, F. S. Tsung, R. Viskup, B. R. Walton, and K. Krushelnick, Nature (London) 431, 535 (2004)

${ }^{8}$ J. Faure, Y. Glinec, A. Pukhov, S. Kiselev, S. Gordienko, E. Lefebvre, J. P. Rousseau, F. Burgy, and V. Malka, Nature (London) 431, 541 (2004).

${ }^{9}$ C. G. R. Geddes, C. Toth, J. v. Tilborg, E. Esarey, C. B. Schroeder, D. Bruhwilder, C. Nieter, J. Cary, and W. P. Leemans, Nature (London) 431, 538 (2004).

${ }^{10}$ A. Pukhov and J. Meyer-ter Vehn, Appl. Phys. B: Lasers Opt. 74, 355 (2002).

${ }^{11}$ G.-Z. Sun, E. Ott, Y. C. Lee, and P. Guzdar, Phys. Fluids 30, 526 (1987).

${ }^{12}$ J. B. Rosenzweig, B. Breizman, T. Katsouleas, and J. J. Su, Phys. Rev. A 44, R6189 (1991).

${ }^{13}$ R. M. G. M. Trines and P. A. Norreys, Phys. Plasmas 13, 123102 (2006).

${ }^{14}$ I. Kostyukov, A. Pukhov, and S. Kiselev, Phys. Plasmas 11, 5256 (2004).

${ }^{15}$ D. Gordon, K. C. Tzeng, C. E. Clayton, A. E. Dangor, V. Malka, K. A.
Marsh, A. Modena, W. B. Mori, P. Muggli, Z. Najmudin, D. Neely, C. Danson, and C. Joshi, Phys. Rev. Lett. 80, 2133 (1998).

${ }^{16}$ V. Malka, S. Fritzler, E. Lefebvre, M. M. Aleonard, F. Burgy, J. P. Chambaret, J. F. Chemin, K. Krushelnick, G. Malka, S. P. D. Mangles, Z. Najmudin, M. Pittman, J.-P. Rousseau, J.-N. Scheurer, B. Walton, and A. E. Dangor, Science 298, 1596 (2002).

${ }^{17}$ B. Hidding, K. U. Amthor, B. Liesfeld, H. Schwoerer, S. Karsch, M. Geissler, L. Veisz, K. Schmid, J. G. Gallacher, S. P. Jamison, D. Jaroszynski, G. Pretzler, and R. Sauerbrey, Phys. Rev. Lett. 96, 105004 (2006).

${ }^{18}$ M. Mori, M. Kando, I. Daito, H. Kotaki, Y. Hayashi, A. Yamazaki, K. Ogura, A. Sagisaka, J. Koga, K. Nakajima et al., Phys. Lett. A 356, 146 (2006).

${ }^{19}$ E. Esarey, P. Sprangle, J. Krall, and A. Ting, IEEE Trans. Plasma Sci. 24, 252 (1996).

${ }^{20}$ M. Geissler, Ph.D. thesis, TU Wien, 2000.

${ }^{21}$ P. Sprangle, J. Krall, and E. Esarey, Phys. Rev. Lett. 73, 3544 (1994).

${ }^{22}$ C. Ren and W. B. Mori, Phys. Plasmas 8, 3118 (2001).

${ }^{23}$ A. Pukhov, S. Gordienko, S. Kiselev, and I. Kostyukov, Plasma Phys. Controlled Fusion 46, B179 (2004).

${ }^{24}$ S. Gordienko and A. Pukhov, Phys. Plasmas 12, 043109 (2005).

${ }^{25}$ A. Pukhov and S. Gordienko, Philos. Trans. R. Soc. London, Ser. A 364, 623 (2006).

${ }^{26}$ W. Lu, M. Tzoufras, C. Joshi, F. S. Tsung, W. B. Mori, J. Vieira, R. A. Fonseca, and L. O. Silva, Phys. Rev. ST Accel. Beams 10, 061301 (2007).

${ }^{27}$ Y. Glinec, J. Faure, A. Norlin, A. Pukhov, and V. Malka, Phys. Rev. Lett. 98, 194801 (2007).

${ }^{28}$ Y. Glinec personal communication (2007).

${ }^{29}$ A. Oguchi, A. Zhidkov, K. Takano, E. Hotta, K. Nemoto, and K. Nakajima, Phys. Plasmas 15, 043102 (2008).

${ }^{30}$ S. P. D. Mangles, A. G. R. Thomas, M. C. Kaluza, O. Lundh, F. Lindau, A. Persson, F. S. Tsung, Z. Najmudin, W. B. Mori, C.-G. Wahlström et al., Phys. Rev. Lett. 96, 215001 (2006).

${ }^{31}$ K. Németh, B. Shen, Y. Li, H. Shang, R. Crowell, K. C. Harkay, and J. R. Cary, Phys. Rev. Lett. 100, 095002 (2008).

${ }^{32}$ K. A. Tanaka, T. Yabuuchi, T. Sato, R. Kodama, Y. Kitagawa, T. Takahashi, T. Ikeda, Y. Honda, and S. Okuda, Rev. Sci. Instrum. 76, 013507 (2005).

${ }^{33}$ P. M. Woodward, J. Inst. Electr. Eng., Part 3 93, 1554 (1946).

${ }^{34}$ J. D. Lawson, IEEE Trans. Nucl. Sci. NS-26, 4217 (1979). 